\title{
ESTUDIOS CROMOSOMICOS EN ESPECIES DE TURNERA (TURNERACEAE)
}

\author{
por VIVIANA G. SOLIS NEFFA' y AVELIANO FERNANDEZ ${ }^{2}$
}

\section{Summary}

In the present paper the chromosome numbers of 5 species and 1 variety of Turnera are reported for the first time. Previous reports on 5 taxa are confirmed. The karyotypes of 16 taxa of Turnera are described for the first time. The results of the chromosome analysis were the following: $T$. weddelliana, $2 \mathrm{n}=2 \mathrm{x}=14=12 \mathrm{~m}+2 \mathrm{sm} ;$. pumilea, $2 \mathrm{n}=2 \mathrm{x}=14=10 \mathrm{~m}+4 \mathrm{sm}$; T. hassleriana, $2 \mathrm{n}=2 \mathrm{x}=14=12 \mathrm{~m}+2 \mathrm{sm} ;$. . chamaedryfolia, $2 \mathrm{n}=2 \mathrm{x}=26=16 \mathrm{~m}$ $+8 \mathrm{sm}+2 \mathrm{st} ; T$. caerulea, $2 \mathrm{n}=2 \mathrm{x}=10=8 \mathrm{~m}+2 \mathrm{sm} ; T$. concinna, $2 \mathrm{n}=2 \mathrm{x}=10=8 \mathrm{~m}+2 \mathrm{sm} ;$ T. krapovickasii, $2 \mathrm{n}=2 \mathrm{x}=10=8 \mathrm{~m}+2 \mathrm{sm} ; T$. grandiflora, $2 \mathrm{n}=8 \mathrm{x}=40=32 \mathrm{~m}+8 \mathrm{sm} ;$. subulata, $2 \mathrm{n}=2 \mathrm{x}=10=8 \mathrm{~m}+2 \mathrm{sm} ; T$. grandidentata, $2 \mathrm{n}=4 \mathrm{x}=20=18 \mathrm{~m}+2 \mathrm{sm} ; T$. angustifolia, $2 \mathrm{n}=6 \mathrm{x}=30=26 \mathrm{~m}+$ $4 \mathrm{sm} ;$ T. orientalis, $2 \mathrm{n}=6 \mathrm{x}=30=26 \mathrm{~m}+4 \mathrm{sm} ;$ T. ulmifolia, $2 \mathrm{n}=6 \mathrm{x}=30=24 \mathrm{~m}$ $+6 \mathrm{sm} ;$ T. velutina, $2 \mathrm{n}=6 \mathrm{x}=30=28 \mathrm{~m}+2 \mathrm{sm} ;$ T. aurelioi, $2 \mathrm{n}=8 \mathrm{x}=40=36 \mathrm{~m}+$ $4 \mathrm{sm}$ and $T$. cuneiformis, $2 \mathrm{n}=8 \mathrm{x}=40=36 \mathrm{~m}+4 \mathrm{sm}$.

The chromosomes are of small size. The species of the series Salicifoliae and Leiocarpae $(\mathrm{x}=7)$ have chromosomes which are smaller than those of the series Canaligerae $(\mathrm{x}=5)$ and Papilliferae $(\mathrm{x}=13)$.

All species studied have symmetrical karyotypes. The scatter diagram shows that species with different basic numbers are perfectly separated. T. chamaedryfolia $(\mathrm{x}=13)$ of the series Papilliferae is the most asymmetrical while the species of the series Canaligerae are the most symmetrical. Species with $\mathrm{x}=7$ occupy a central position in the diagram. 
The diploid species of Canaligerae studied had the same basic karyotype: $8 \mathrm{~m}+2 \mathrm{sm}$, which can be considered as the fundamental karyotype of the series. The information obtained concerning karyotype analysis of these species support their taxonomic position.

\section{Introducción}

El génerc Turnera es el más numeroso y el más estudiado citológicamente dentro de la familia. Se conoce el número cromosómico de unas 25 especies (Hamel, 1965; Barrett, 1978; Barrett y Shore, 1980; Raman y Kesavan, 1964; Arbo y Fernández, 1983 y Fernández, 1987) pertenecientes a seis series diferentes. El número básico más frecuente es $\mathrm{x}=7$, encontrándose $\mathrm{x}=13$ en la serie monotípica Papilliferae y $\mathrm{x}=5$ en la serie Canaligerae. Se han hallado también varios niveles de ploidía, desde $2 x$ hasta 8x en la serie Canaligerae (Raman \& Kesavan, 1964; Barrett, 1978; Arbo \& Fernández, 1983; Shore y Barrett, 1985; Fernández, 1987) y desde 2x hasta 10x en la serie Leiocarpae (Fernández, 1987). Los trabajos de Fernández (1987) sobre la meiosis en poliploides indican que hay auto- y alopoliploides.

En este trabajo se estudian cromosómicamente 21 especies y una variedad, se presenta el primer recuento para T. trigona, T. arcuata, $T$. candida, T. velutina, T. ulmifolia var. acuta y T. cuneiformis, recuentos de nuevas accesiones de T. pumilea, T. scabra, T. hermannioides, $T$. orientalis y $T$. grandiflora, y se describen por primera vez los cariotipos de 16 especies pertenecientes a 4 series.

\section{Materiales y métodos}

En la Tabla 1 se cita el material utilizado. Los ejemplares testigos se encuentran depositados en el Herbario CTES.

Se emplearon meristemas de raíces pretratados con 8-oxiquinoleína $0,002 \mathrm{M}$ durante 3 horas a temperatura ambiente, fijados en 5 partes de alcohol etílico y una parte de ácido láctico (Fernández, 1973) durante aproximadamente 12 horas y conservadas en alcohol $70 \%$ a $4{ }^{\circ} \mathrm{C}$. Las raí- 
Tabla 1. Especies estudiadas. * Primer recuento, ** recuento de nuevas accesiones.

\begin{tabular}{lll}
\hline Especie & $2 \mathrm{n}$ & Coleccionista y procedencia \\
\hline
\end{tabular}

\section{Turnera}

Serie Salicifoliae

T. weddelliana Urban \& Rolfe $14 \quad$ Schinini 21213, Paraguay, Chaco, Col. San Alfredo.

Serie Leiocarpae

T. pumilia $\mathrm{L}$.

14 Krapovickas 37189, Brasil, MA,

Timon, ca. Teresina.

“

14** Arbo 4079, Brasil, MG, ca.

Diamantina.

T. hassleriana Urban

14 Krapovickas 36444, Bolivia, dep.

S. Cruz, ca. Santiago.

T. trigona Urban

14* Arbo et al. 3326, Brasil, GO,

Serra dos Cristais, ca. Cristalina.

Serie Papilliferae

T. chamaedryfolia Cambess.

26 Noblick 3175, Brasil, BA, Feira de Santana.

Serie Canaligerae

T. caerulea DC.

10 Krapovickas 38740, Brasil, PI, Bom Jesus.

“

10 Krapovickas 37914, Brasil, GO, BR-153, $18 \mathrm{~km} \mathrm{~N}$ de Colinas.

T. scabra Millspaugh

10** Breedlove 47230, México, Chiapas, Catazajá.

T. concinna Arbo

10 Vanni 213, Paraguay, San Pedro, ca. Col. Guayaibí.

T. krapovickasii Arbo

10 Krapovickas 38858, Bolivia, Tarija, O'Connor, Entre Ríos.

10 Krapovickas 39177, Bolivia, Tarija, Gran Chaco, Villa Montes.

20 Beck 9433, Paraguay, Nueva Asunción, Gral. E. A. Garay, ca. Estigarribia.

20 Krapovickas \& Schinini 36121, Bolivia, Santa Cruz. 


\begin{tabular}{|c|c|c|}
\hline “ & 20 & $\begin{array}{l}\text { Schinini } 19515 \text {, Argentina, Salta, } \\
\text { Anta, El Quebrachal. }\end{array}$ \\
\hline T. subulata Smith & 10 & Arbo 2410, Brasil, MA, Sao Luiz. \\
\hline “ & 20 & $\begin{array}{l}\text { Krapovickas } 37115 \text {, Brasil, AM, } \\
\text { Manaus. }\end{array}$ \\
\hline T. hermannioides Urban & $10^{* *}$ & $\begin{array}{l}\text { Hatschbach } 56668, \text { Brasil, BA, } \\
\text { Livramento do Brumado. }\end{array}$ \\
\hline T. candida Arbo & $10^{*}$ & $\begin{array}{c}\text { Hatschbach 56713, BA, } \\
\text { Brasil, Rio de Contas. }\end{array}$ \\
\hline T. grandidentata (Urban) & 20 & Schinini 23981, Paraguay, \\
\hline Arbo & & $\begin{array}{l}\text { Cordillera, Cordillera de Altos, } \\
\text { Col. Tobaty. }\end{array}$ \\
\hline T. arcuata Urban & $20^{*}$ & $\begin{array}{l}\text { Arbo et al. } 3313 \text {, Brasil, GO, } \\
\text { Morro do Padre, ca. Cristalina. }\end{array}$ \\
\hline T. angustifolia Miller & 30 & $\begin{array}{l}\text { Barrett } 1254 \text {, Costa Rica, } \\
\text { Guanacaste, Cañas. }\end{array}$ \\
\hline T. orientalis (Urban) & 30 & Schinini \& Daviña 24717 \\
\hline Arbo & & $\begin{array}{l}\text { Argentina, Misiones, San Ignacio, } \\
\text { Peñón de Reina Victoria, } \\
\text { Río Paraná. }\end{array}$ \\
\hline “ & $30 * *$ & $\begin{array}{l}\text { Schinini } 27412 \text {, Paraguay, Alto } \\
\text { Paraná, ca. Hernandarias, Ea. } \\
\text { Santa Elena. }\end{array}$ \\
\hline “" & $30^{* *}$ & $\begin{array}{l}\text { Arbo 5156, Brasil, MG, ca. } \\
\text { Diamantina. }\end{array}$ \\
\hline T. ulmifolia L. sensu stricto & 30 & $\begin{array}{l}\text { Arbo } 2698 \text {, cult. Corrientes, } \\
\text { semillas procedentes de Miami, } \\
\text { Florida, USA. }\end{array}$ \\
\hline $\begin{array}{l}\text { T. ulmifolia var. acuta } \\
\text { (Spreng.) Urban }\end{array}$ & $30 *$ & $\begin{array}{l}\text { Correll 40638, Bahamas, Pelican } \\
\text { Lake. }\end{array}$ \\
\hline T. velutina Presl & $30 *$ & $\begin{array}{l}\text { Koch \& Fryxell 78341, México, } \\
\text { Oaxaca, Santo Domingo. }\end{array}$ \\
\hline T. aurelioi Arbo & 40 & $\begin{array}{l}\text { Schinini } 23860 \text {, cult. Corrientes, } \\
\text { semillas procedentes del } \\
\text { Paraguay, ca. Emboscada. }\end{array}$ \\
\hline T. cuneiformis Poiret & $40^{*}$ & $\begin{array}{l}\text { Arbo } 4985, \text { Brasil. MG, } \\
\text { Conceicao do Mato Dentro. }\end{array}$ \\
\hline$T$. grandiflora (Urban) Arbo & $40 * *$ & $\begin{array}{l}\text { Klassen 17, Paraguay, Chaco, } \\
\text { Col. Fernheim, Laguna Porá. }\end{array}$ \\
\hline
\end{tabular}


ces se tiñeron usando la técnica de Feulgen. Los preparados permanentes se hicieron con la técnica de Bowen (1956).

La nomenclatura utilizada para la descripción de los cariotipos es la propuesta por Levan et al. (1964). En los idiogramas los cromosomas fueron ordenados del siguiente modo: primero los metacéntricos, luego los submetacéntricos y en último término los subtelocéntricos y a su vez de mayor a menor.

Se estimaron los siguientes parámetros para caracterizar numéricamente los cariotipos:

- Longitud total del cariotipo

- Volumen cromosómico $=$ long. total $\times 2 \times 3,14 \times(\text { ancho/4 })^{2}$

La estimación de la asimetría de los cariotipos se realizó por el índice centromérico y también por el método propuesto por Romero Zarco (1986): $\mathrm{A}_{1}=$ índice de asimetría intracromosómica y $\mathrm{A}_{2}=$ índice de asimetría intercromosómica, los que se representan gráficamente en un diagrama de dispersión.

\section{Resultados}

Los resultados se muestran en la Tabla 2 y en las Figuras 3, 4 y 5. A continuación se describen las características más destacadas de cada uno de los cariotipos.

\section{Serie Salicifoliae}

T. weddelliana. $2 \mathrm{n}=2 \mathrm{x}=14$ (Fig. 1A). Posee 6 pares metacéntricos y un par submetacéntrico (Fig. 3A). Tiene los cromosomas más pequeños (1.12 micras) entre todas las especies analizadas hasta el momento.

\section{Serie Leiocarpae}

T. pumilea. $2 \mathrm{n}=2 \mathrm{x}=14$ (Fig. 1B). Posee cinco pares metacéntricos $\mathrm{y}$ dos pares submetacéntricos. Macrosatélite en el brazo corto del par dos (Fig. 3 B). Los cromosomas son también muy pequeños (1,24 micras). 
Tabla 2. Número cromosómico, nivel de ploidía, fórmula cariotípica, longitud total (LT), longitud cromosómica media (LM), volumen cromosómico (VC), índice centromérico (IC) ,índice de asimetría intracromosómica $\left(\mathrm{A}_{1}\right)$ e intercromosómica $\left(\mathrm{A}_{2}\right)$.

\begin{tabular}{llllllllllll}
\hline Especie & $2 n$ & $X$ & Cariotipo & LT & LM & VC & IC & $A_{1}$ & $A_{2}$ \\
& & & $\mu$ & $\mu^{3}$ & & & &
\end{tabular}

Serie Salicifoliae
T. weddelliana
$14 \quad 212 m+2 s m$
15,72
1,12
2,96
$42,29 \quad 0,28 \quad 0,18$

Serie Leiocarpae

$\begin{array}{llllllllll}\text { T. pumilea } & 14 & 2 & 10 \mathrm{~m}+4 \mathrm{sm} & 17,44 & 1,24 & 4,38 & 42,82 & 0,26 & 0,21 \\ \text { T. hassleriana } & 14 & 2 & 12 \mathrm{~m}+2 \mathrm{sm} & 25,68 & 1,83 & 8,06 & 43,94 & 0,22 & 0,25\end{array}$

Serie Papilliferae

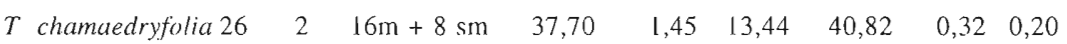

$$
+2 s t
$$

Serie Canaligerae

$\begin{array}{lrrrrrrrrr}T \text { caerulea } & 10 & 2 & 8 \mathrm{~m}+2 \mathrm{sm} & 23,84 & 2,53 & 10,47 & 44,00 & 0,23 & 0,18 \\ T . \text { concinna } & 10 & 2 & 8 \mathrm{~m}+2 \mathrm{sm} & 25,30 & 2,52 & 9,53 & 44,25 & 0,21 & 0,16 \\ T \text { krapovickasii } & 10 & 2 & 8 \mathrm{~m}+2 \mathrm{sm} & 22,82 & 2,28 & 6,30 & 43,75 & 0,22 & 0,14 \\ \text { T. krapovickasii } & 20 & 4 & 16 \mathrm{~m}+4 \mathrm{sm} & 40,32 & 2,02 & 13,25 & 44,99 & 0,19 & 0,13 \\ T \text { grandiflora } & 40 & 8 & 32 \mathrm{~m}+8 \mathrm{sm} & 73,20 & 1,83 & 22,98 & 44,58 & 0,21 & 0,24 \\ T . \text { subulata } & 10 & 2 & 8 \mathrm{~m}+2 \mathrm{sm} & 17,10 & 1,71 & 4,29 & 45,50 & 0,17 & 0,13 \\ T . \text { subulata } & 20 & 4 & 16 \mathrm{~m}+2 \mathrm{sm} & 34,96 & 1,74 & 6,58 & 45,63 & 0,17 & 0,22 \\ T . \text { grandidentata } & 20 & 4 & 18 \mathrm{~m}+2 \mathrm{sm} & 41,28 & 2,06 & 18,14 & 45,58 & 0,16 & 0,20 \\ T . \text { angustifolia } & 30 & 6 & 26 \mathrm{~m}+4 \mathrm{sm} & 56,56 & 1,88 & 17,75 & 44,44 & 0,21 & 0,14 \\ T . \text { orientalis } & 30 & 6 & 26 \mathrm{~m}+4 \mathrm{sm} & 75,52 & 2,51 & 20,80 & 44,33 & 0,15 & 0,13 \\ T \text { ulmifolia } & 30 & 6 & 24 \mathrm{~m}+6 \mathrm{sm} & 69,66 & 2,32 & 26,24 & 42,57 & 0,23 & 0,13 \\ T \text { velutina } & 30 & 6 & 28 \mathrm{~m}+2 \mathrm{sm} & 71,52 & 2,38 & 25,78 & 44,98 & 0,18 & 0,14 \\ T . \text { aurelioi } & 40 & 8 & 36 \mathrm{~m}+4 \mathrm{sm} & 88,58 & 2,21 & 26,51 & 44,58 & 0,15 & 0,24 \\ T \text { cuneiformis } & 40 & 8 & 36 \mathrm{~m}+4 \mathrm{sm} & 54,40 & 1,36 & 20,49 & 44,78 & 0,20 & 0,14\end{array}$

Fig. 1.- Metafases mitóticas. A, T. weddelliana; B, T. pumilea; C, T. hassleriana; D, T. chamaedryfolia; E, T. grandiflora; F, T. caerulea; G-H, T. krapovickasii; I, T. concinna; J-K. T. subulata. Escala $=10$ micras. 

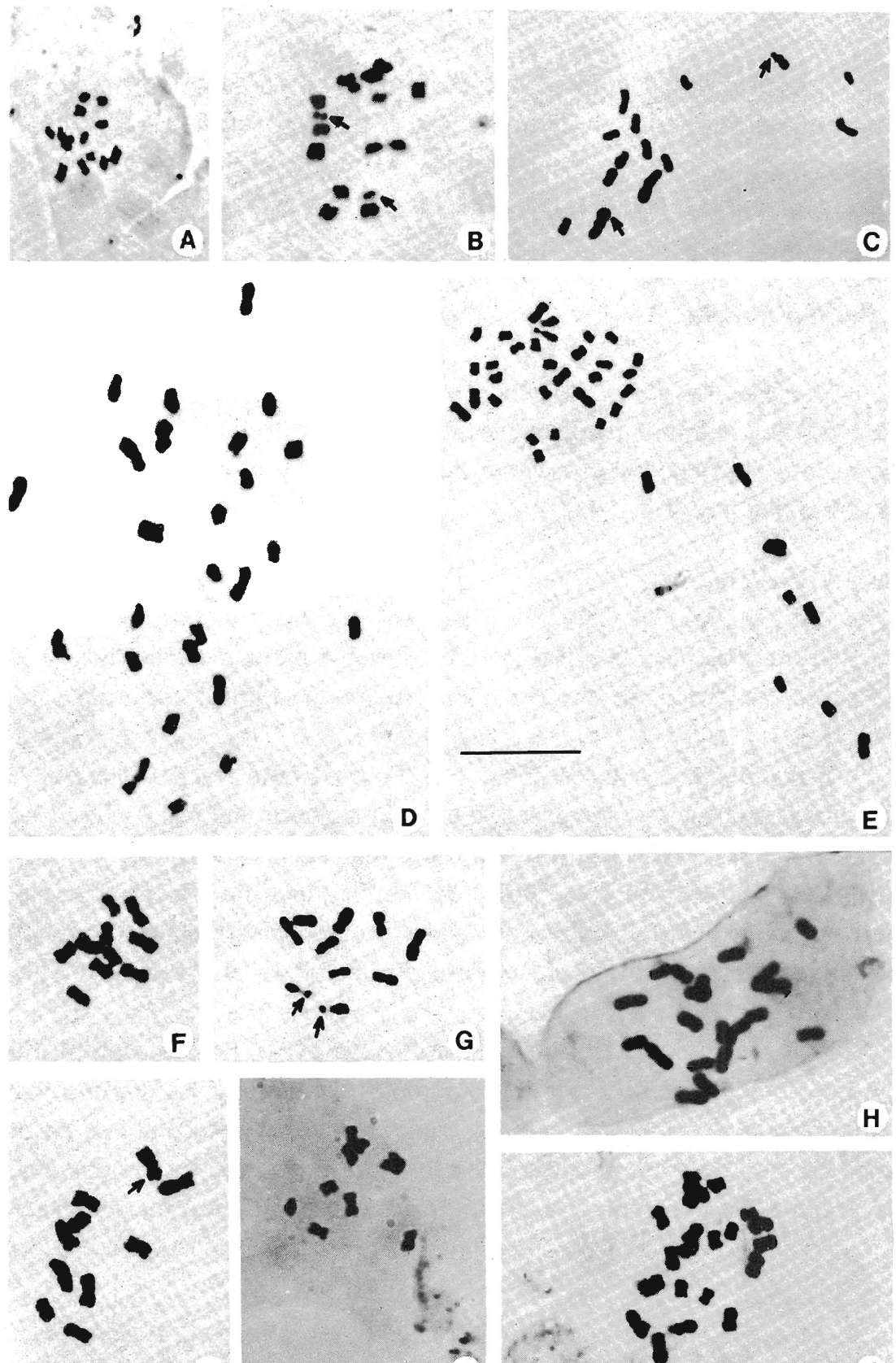

G

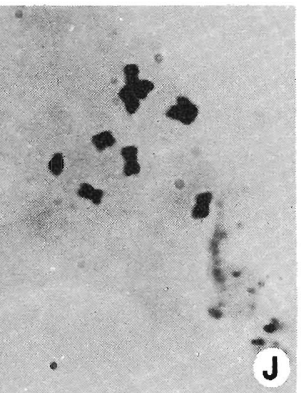

H 
T. hassleriana. Citotipos diploide $2 n=2 x=14$ y tetraploide $2 n=4 x=28$. Sólo se analizó el citotipo diploide $2 n=2 x=14$ (Fig. 1C). Posee seis pares metacéntricos y un par submetacéntrico. Se observaron dos pares con satélites. El par 1 con macrosatélite en el brazo largo y el par 3 con microsatélite en el brazo corto (Fig. $3 \mathrm{C}$ ).

\section{Serie Papilliferae}

T. chamaedryfolia. $2 \mathrm{n}=2 \mathrm{x}=26$ (Fig. 1D). Es la más asimétrica (IC $=40,82$ ), presenta 8 pares metacéntricos, 4 pares submetacéntricos y un par subtelocéntrico. Se observaron dos pares (10 y 11) con microsatélite en el brazo corto (Fig. 3 D).

\section{Serie Canaligerae}

T. caerulea. $2 \mathrm{n}=2 \mathrm{x}=10$ (Fig. $1 \mathrm{~F}$ ). Posee 4 pares metacéntricos y un par submetacéntrico. Se observa microsatélite en el brazo corto del par 1 (Fig. 4 A).

T. concinna. $2 \mathrm{n}=2 \mathrm{x}=10$ (Fig. $1 \mathrm{I}$ ). Posee 4 pares metacéntricos y 1 par submetacéntrico y macrosatélite en el brazo corto del par 2 (Fig. 4 B).

T. krapovickasii. Citotipos diploide, $2 \mathrm{n}=2 \mathrm{x}=10$ (Fig. $1 \mathrm{G}$ ) y tetraploide, $2 n=4 x=20$ (Fig. $1 \mathrm{H}$ ). Se confeccionaron los cariotipos de ambos citotipos. En el diploide se observaron 4 pares metacéntricos y 1 par submetacéntrico, el par dos con macrosatélite en el brazo corto (Fig. $4 \mathrm{C})$.

T. grandiflora. Citotipos diploide, $2 \mathrm{n}=2 \mathrm{x}=10$ y auto-octoploide $2 n=8 x=40$ (Fig. 1 E). El citotipo octoploide, que posee 32 cromosomas metacéntricos distribuidos en cuatro grupos y 8 submetacéntricos en un único grupo. Se vieron microsatélites en el brazo corto de los cromosomas del grupo 3. Se observaron hasta un máximo de 3 cromosomas con saté-

Fig. 2.- Metafases mitóticas. A, T. grandidentata; B, T. orientalis; C, T. angustifolia; D, T. ulmifolia; E, T. velutina; F, T. aurelioi; $\mathrm{G}, T$. cuneiformis. Escala $=10$ micras. 

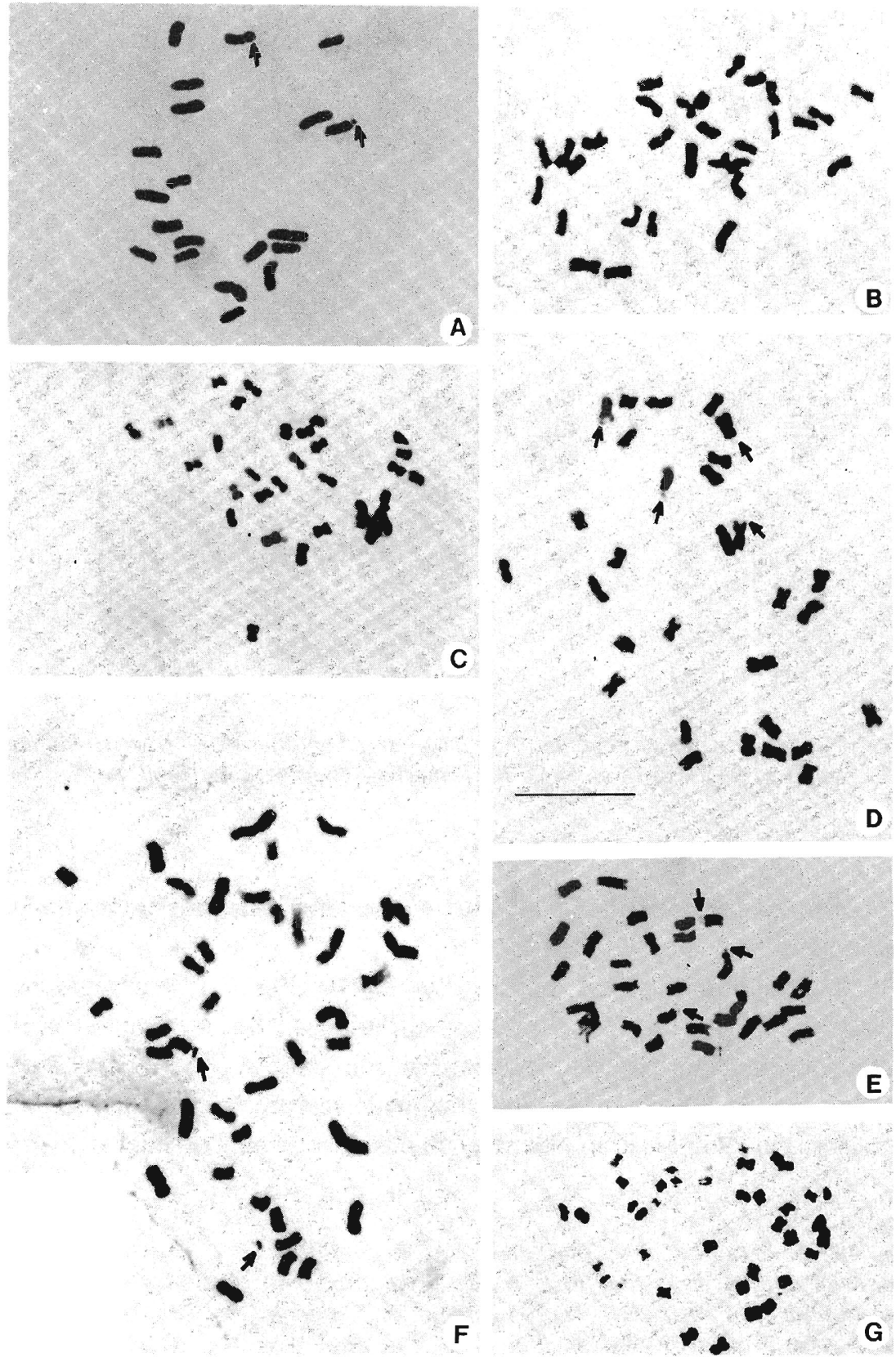

A 

$\begin{array}{lllllllllllll}1 & 2 & 3 & 4 & 5 & 6 & 7 & 8 & 9 & 10 & 11 & 12 & 13\end{array}$

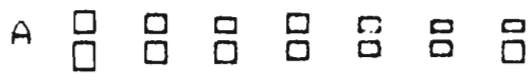
m
$5 m$

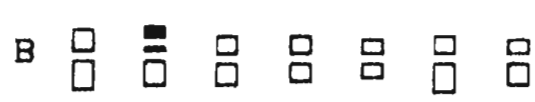
m $\quad$ sm

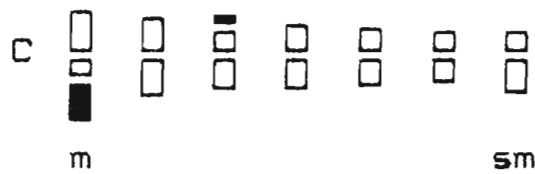

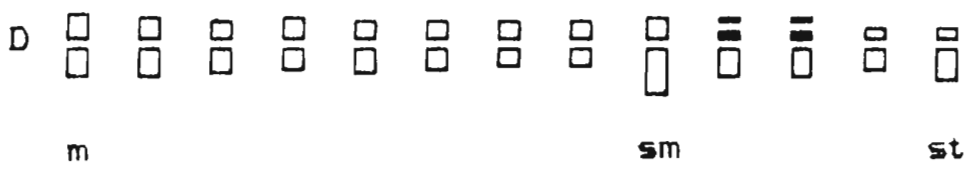

Fig. 3.- Idiogramas, Series Salicifoliae, Leiocarpae y Papilliferae. A, T. weddelliana; B, T. pumilea; C, T. hassleriana; D, T. chamaedryfolia. Escala $=10$ micras.

lite por metafase analizada. En la figura $4 \mathrm{D}$ se representan los cromosomas correspondientes a un genoma.

T. subulata. Citotipos diploide, $2 \mathrm{n}=2 \mathrm{x}=10$ (Fig. $1 \mathrm{~J}$ ) y tetraploide, $2 n=2 x=20$ (Fig. $1 K$ ). En el citotipo diploide se observaron 4 pares metacéntricos y un par submetacéntrico con satélite (Fig. 4 E). En el citotipo tetraploide se observaron solamente metacéntricos. En algunas metafases del citotipo tetraploide se pudo observar un par con microsatélite.

Fig. 4.- Idiogramas, Serie Canaligerae. A, T. caerulea; B, $T$. concinna; C, $T$. krapovickasii; D, T. grandiflora; E, T. subulata; F, T. grandidentata; G, T. angustifolia; H. T. orientalis; I, T. ulmifolia; J, T. velutina; K, T. aurelioi; L, T. cuneiformis. Escala $=10$ micras. 
$\begin{array}{llllllllllllllllllll}1 & 2 & 3 & 4 & 5 & 6 & 7 & 8 & 9 & 10 & 11 & 12 & 13 & 14 & 15 & 10 & 17 & 18 & 19 & 20\end{array}$ 40

$m$

$5 m$

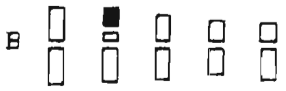

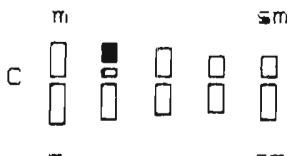

$\begin{array}{llllll}m & & & & 5 m \\ \square & \square & 0 & \square & \square & \square \\ \square & \square & \square & \square\end{array}$

E $\begin{array}{llllll}m & & & & \text { sm } \\ \square & \square & \square & \square & \bar{a} \\ \square & \square & \square & \square & \square\end{array}$

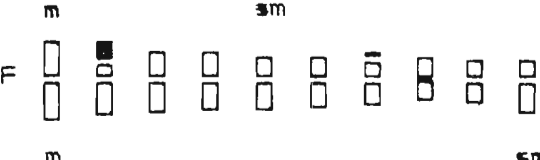

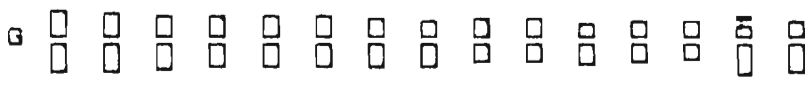

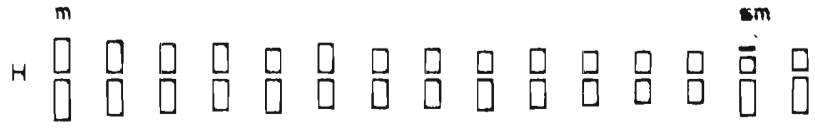

m

$\begin{array}{llllllllllllllll} & \square & \square & \square & \square & \square & \square & \bar{\square} & \square & \square & \square & \square & \square & \bar{b} & \square & \square \\ & \square & \square & \square & \square & \square & \square & \square & \square & \square & \square & \square & \square & \square & \square & \square\end{array}$

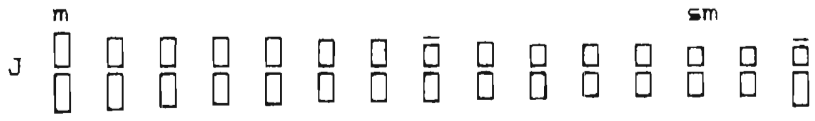

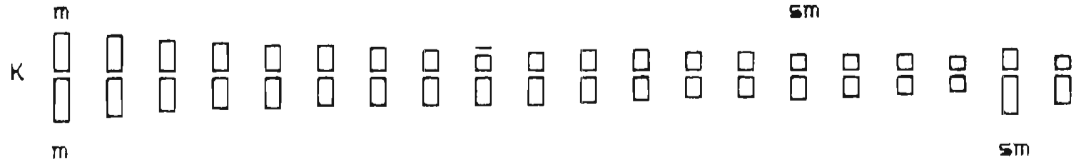

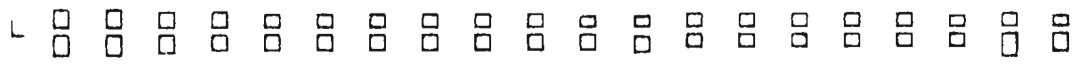


T. grandidentata. Alotetraploide, $2 \mathrm{n}=4 \mathrm{x}=20$ (Fig. 2A). Posee 9 pares metacéntricos y un par submetacéntrico. Se observó macrosatélite en el brazo corto del par 2 y microsatélite en el brazo corto del par 7 (Fig. 4 F).

T. angustifolia. Alohexaploide, $2 \mathrm{n}=6 \mathrm{x}=30$ (Fig. 2C). Se observaron 13 pares metacéntricos y dos pares submetacéntricos. El par 14 posee microsatélite en el brazo corto (Fig. $4 \mathrm{G}$ ).

T. orientalis. Alohexaploide, $2 \mathrm{n}=6 \mathrm{x}=30$ (Fig. 2B). Posee 13 pares metacéntricos y dos pares submetacéntricos. Se observó el par 14 con microsatélite en el brazo corto (Fig. $4 \mathrm{H}$ ).

T. ulmifolia. Alohexaploide, $2 \mathrm{n}=6 \mathrm{x}=30$ (Fig. 2 D). Doce de los quince pares son metacéntricos y tres son submetacéntricos. Posee dos pares (7 y 13) con satélite, ambos en el brazo corto (Fig. 4 I).

T. velutina. Alohexaploide, $2 \mathrm{n}=6 \mathrm{x}=30$ (Fig. 2 E). Posee 14 pares metacéntricos y 1 par submetacéntrico, con microsatélite en el brazo corto del par 8 y par 15 (Fig. $4 \mathrm{~J}$ ).

T. aurelioi. Alo-octoploide, $2 \mathrm{n}=8 \mathrm{x}=40$ (Fig. 2 F). Se observaron 18 pares metacéntricos y dos pares submetacéntricos. Con microsatélite en el brazo corto del par 9 (Fig. $4 \mathrm{~K}$ ).

T. cuneiformis. Octoploide, $2 \mathrm{n}=8 \mathrm{x}=40$ (Fig. $2 \mathrm{G}$ ). Presenta 18 pares metacéntricos y 2 submetacéntricos (Fig. 4 L).

\section{Discusión y conclusiones}

Los cromosomas de Turnera corresponden a la categoría de pequeños de acuerdo a la clasificación de Lima de Faría (1980). Las especies de las series Salicifoliae y Leiocarpae $(x=7)$ muestran en general cromosomas más pequeños que las especies de las series Canaligerae $(\mathrm{x}=5)$ y Papilliferae ( $\mathrm{x}=13)$. T. weddelliana de la serie Salicifoliae es la especie que presenta los cromosomas de menor tamaño ( $\mathrm{LM}=1,12$ micras) y $T$. caerulea de la serie Canaligerae los más grandes ( $\mathrm{LM}=2,53$ micras).

Considerando que las especies $\mathrm{x}=5$ serían derivadas de las $\mathrm{x}=7$ (Fernández, 1987) y que en general poseen cromosomas más grandes, la diferencia en tamaño se explicaría por translocaciones cromosómicas con pérdida de centrómeros originando especies con menor número de 
cromosomas pero de mayor tamaño (en ambos casos la mayoría, metacéntricos).

En general las especies con $x=5, x=7$ y $x=13$, presentan los cariotipos simétricos. En el diagrama de dispersión (Fig. 5) se observa una perfecta separación de las mismas de acuerdo con su número básico. Las especies $\mathrm{x}=7$ ocupan una posición central con respecto a las demás, $T$. chamaedryfolia $(\mathrm{x}=13)$, ocupa la posición extrema. La mayor simetría observada

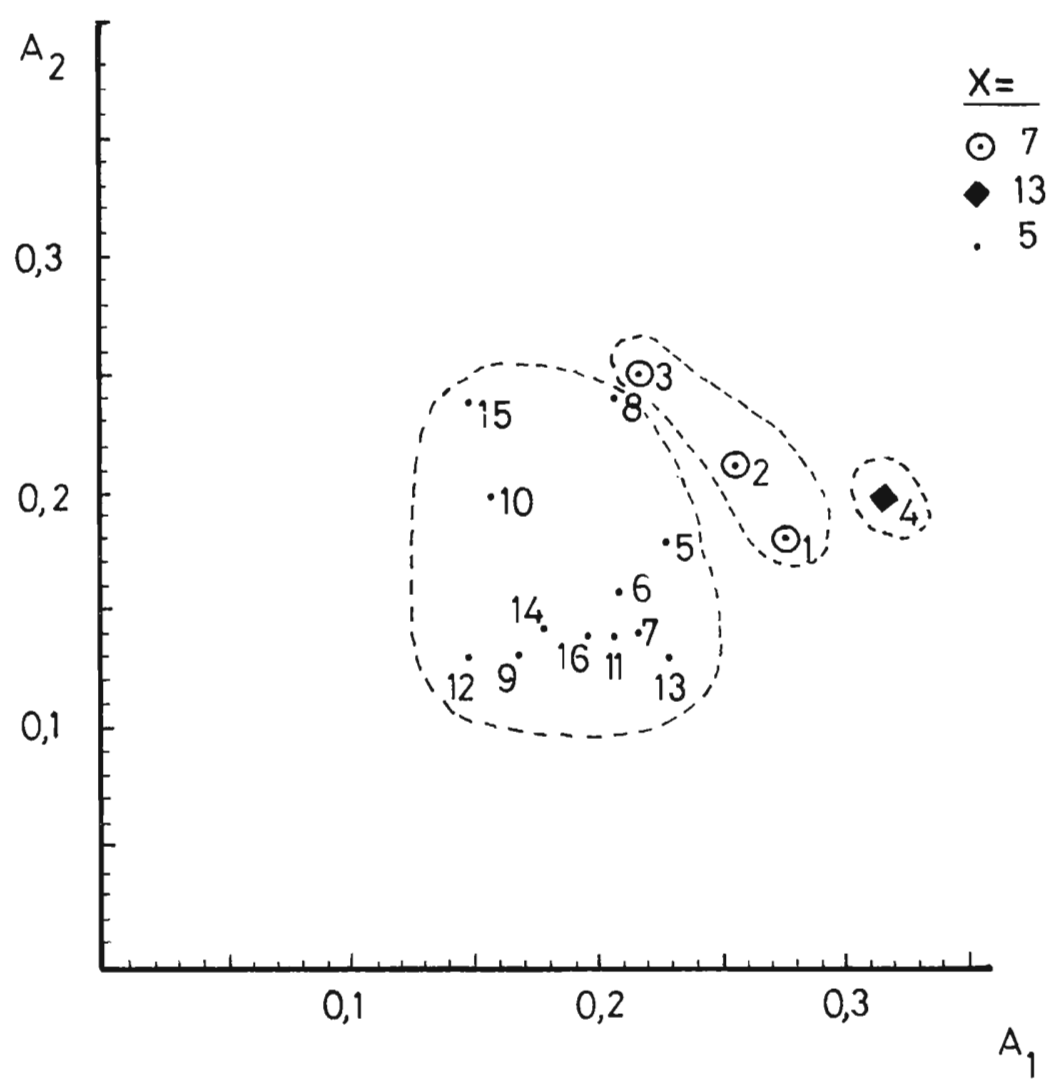

Fig. 5.- Diagrama de dispersión. 1, T. weddelliana; $2, T$. pumilea; 3 , T. hassleriana; 4, T. chamaedryfolia; 5, T. caerulea; 6. T. concinna; 7. T. krapovickasii; 8, T. grandiflora; $9, T$. subulata; $10, T$. grandidentata; 11, T. angustifolia; 12, T. orientalis; 13, T. ulmifolia; 14, T. velutina; 15. T. aurelioi; 16, T. cuneiformis. 
en las especies de la serie Canaligerae $(\mathrm{x}=5)$, está de acuerdo con su número básico derivado (Fernández, 1987) y representaría una tendencia secundaria en la evolución cromosómica (Stebbins, 1971).

Las especies de la serie Canaligerae se reunen en dos grupos que se diferencian por sus semillas (Fernández y Arbo, 1989). Uno de los grupos constituye el complejo T. ulmifolia que fue descripto por Urban (1883) con más de 10 variedades. Shore y Barrett (1985) hicieron un estudio numérico sobre la diferenciación morfológica en 6 taxones del mencionado complejo; los resultados apoyan la separación del mismo en varias especies. La mayor parte de las variedades de T. ulmifolia se reconocen actualmente como especies independientes (Backer, 1951; Arbo, 1985). Los datos citológicos obtenidos por Fernández (1987), Fernández y Arbo (1989, 1990, 1993) y los del análisis de los cariotipos apoyan dicha separación.

En la serie Canaligerae existen varios niveles de ploidía, desde $2 \mathrm{x}$ hasta $8 x$. Todas las especies diploides $2 n=2 x=10$ analizadas muestran un cariotipo básico haploide $4 \mathrm{~m}+1 \mathrm{sm}$, que podría considerarse el cariotipo fundamental de la serie. Aunque T. caerulea, T. concinna, T. krapovickasii y T. subulata poseen la misma fórmula cariotípica se observan diferencias ya sea en la longitud total del complemento, variación intercromosómica $\left(\mathrm{A}_{2}\right)$ y en el tipo y ubicación de los satélites. De T. grandiflora se analizó el auto-octoploide, se encontró que en su cariotipo está repetido 8 veces el cariotipo fundamental. En la meiosis se han observado octovalentes (Fernández, 1987).

En la Tabla 2 se observa que las especies de flores blanco-azuladas (T. caerulea y $T$. grandiflora) presentan valores más altos de $\mathrm{A}_{2}$ que las especies de flores amarillas (T. krapovickasii, $T$. concinna y $T$. subulata). Tanto las especies de flores blanco-azuladas como las de flores amarillas constituyen grupos muy afines entre sí (Fernández y Arbo, 1989).

Con respecto a estas últimas, se observa que los cariotipos de T. krapovickasii y $T$. concinna son los más similares, por la fórmula cariotípica y por la presencia de macrosatélite en el mismo par (Fig. 4, B-C). De estas dos especies, $T$. concinna es la que presenta mayor volúmen cromosómico y longitud total del genoma. Los valores de A son similares para ambas, pero $T$. krapovickasii posee menor variación inter- 
cromosómica (Tabla 2). El cariotipo de T. subulata (Fig. 4E) se distingue por la presencia de un par de cromosomas con microsatélite. De estas tres especies, $T$. subulata es la que posee los cromosomas más pequeños y con menor volumen y el cariotipo más simétrico. Todas estas características señalarían a $T$. subulata como la especie con el cariotipo menos evolucionado y $T$. concinna el más asimétrico y el más evolucionado. Fernández y Arbo (1989) consideran a T. subulata la especie más primitiva, a partir de la cual se habrían diferenciado $T$. scabra y $T$. krapovickasii; a su vez, $T$. concinna probablemente habría derivado de $T$. krapovickasii. Conforme a ello, T. krapovickasii habría adquirido en el transcurso de la evolución mayor tamaño de cromosomas.

En el caso de T. subulata y T. krapovickasii que presentan citotipos diploide y autotetraploide, se observa que en los diploides los cromosomas son de mayor longitud que en los tetraploides, pero de menor simetría ya que los cromosomas submetacéntricos que se observan en los primeros tienden a hacerse metacéntricos en los tetraploides. De acuerdo con estos resultados en los tetraploides se habrían producido reordenamientos cromosómicos.

T. grandidentata, $T$. orientalis y $T$. aurelioi, serían alopoliploides segmentarios (Fernández y Arbo, 1990 y 1993), probablemente las otras especies poliploides analizadas en este trabajo sean también del mismo tipo.

T. grandidentata es una especie tetraploide en donde los cromosomas se agrupan de a pares con la presencia de un par con microsatélite y un par con macrosatélite.

De las cuatro especies hexaploides, $T$. angustifolia y $T$. orientalis poseen la misma fórmula cariotípica $2 n=6 x=26 m+4 s m$, se diferencian en el tamaño de los cromosomas, T. orientalis posee 2,51 micras de promedio por cromosoma, mientras que $T$. angustifolia posee 1,88 micras. También difieren en los valores de $\mathrm{A}_{1}$ y $\mathrm{A}_{2}$.

Las otras dos especies hexaploides, T. ulmifolia y $T$. velutina se diferencian de las dos anteriores y entre sí porque la primera especie posee $2 n=6 x=24 m+6 s m$ y la segunda $2 n=6 x=28 m+2 s m$. Además estas especies poseen dos pares de cromosomas nucleolares. 
Entre las cuatro especies hexaploides las más similares exomorfológicamente son $T$. ulmifolia y $T$. angustifolia, que se diferencian entre sí por la fórmula cariotípica y por el número y posición de los satélites.

$T$. aurelioi y $T$. cuneiformis son octoploides con la misma fórmula cariotípica $2 n=8 x=36 m+4 s m$, pero en la primera especie se observa un par de cromosomas con satélite, el que no pudo ser detectado en la segunda. T. aurelioi tiene los cromosomas más grandes que T. cuneiformis (Tabla 2).

Las especies hexaploides presentan valores similares de $\mathrm{A}_{1}$ pero difieren en los valores de $A_{2}$. Las especies octoploides presentan más variabilidad para ambos índices.

Fernández y Arbo (1993) sugieren que T. orientalis muy probablemente sea uno de los progenitores de $T$. aurelioi ya que el híbrido obtenido del cruzamiento de ambas especies a pesar de su alto nivel de ploidía $(2 n=7 x=35)$, presenta una sola configuración: $5 I+15$ II. Desde el punto de vista geográfico esto es posible ya que la distribución de $T$. orientalis abarca la de T. aurelioi. Estas especies compartirían seis genomas. La comparación de los cariotipos de estas especies avala esta hipótesis. Los monovalentes observados en la meiosis son grandes y probablemente correspondan a los cromosomas más grandes observados en el cariotipo de T. aurelioi. Estos cromosomas serían los que dan la diferencia en los valores de $\mathrm{A}_{2}$ observados en la Tabla 2 , ya que los valores de $\mathrm{A}_{1}$ son semejantes.

\section{Agradecimientos}

Los autores desean expresar su agradecimiento a la Lic. María Mercedes Arbo por haber facilitado las semillas y por la identificación del material.

\section{Bibliografía}

Arbo, M. M. 1977. Adenoa, nuevo género americano de Turneraceae. Hickenia. 1(16): 87-92. 
- 1981. Novedades en Turneráceas de Brasil. Bonplandia 5 (14): 111 122.

- 1985. Notas taxonómicas sobre Turneráceas sudamericanas. Candollea 40: 175-191.

- 1986. Una nueva Turnera del Paraguay. En R. Spichiger \& G. Bocquet, ed., Notulae ad Floram paraquaiensem 5. Candollea 41 (1): 209-210.

Arbo, M.M. \& Fernández, A. Posición taxonómica, citología y palinología de tres niveles de ploidía de Turnera subulata Smith. Bonplandia 5 (23): 211-226.

- 1987. Cruzamientos intra e interespecíficos en Turnera, Serie Canaligerae. Bonplandia 6(1): 23-38.

Backer, C.A. 1951. Turneraceae. Flora Malesiana Ser. I, 4: 235-238.

Barrett, S.C.H. 1978. Heterostyly in a tropical weed: the reproductive biology of the Turnera ulmifolia complex (Turneraceae). Canad. J. Bot. 56 (15): 1713-1725.

Barrett, S.C.H. \& Shore, J. 1980. Variation in breeding systems in the Turnera ulmifolia complex. Second International Congress of Systematic and Evolutionary Biology, Vancouver, Canada. (Resúmenes, pag. 10).

Bowen, C. C. 1956 . Freezing by liquid carbone dioxide in making slides permanent. Stain Technol. 31: 87-90.

Fernández, A. 1973. El ácido láctico como fijador cromosómico. Bol. Soc. Argent. Bot. 15 (2-3): 287-290.

- 1987. Estudios cromosómicos en Turnera y Piriqueta (Turneraceae). Bonplandia 6 (1): 1-21.

Fernández, A. \& Arbo, M.M. 1989. Relaciones genómicas entre cuatro especies diploides de Turnera con flores amarillas (Serie Canaligerae). Bonplandia 6 (2): 93-102.

- 1993. Relaciones genómicas entre seis especies de Turnera (Serie Canaligerae) del Paraguay. Candollea 48 (1): 305-318.

Hamel, J. L. 1965. Le noyau et les chromosomes somatiques de Turnera ulmifolia L. Mém. Mus. Natl. Hist. Nat. Sér. B, 16 (1): 3-8. 
Levan, A.; Fredga, K. and A. A. Sandberg. 1964. Nomenclature for centromeric position on chromosomes. Hereditas 52 (2): 201-220.

Lima de Faría, A. 1980. Classification of genes, rearrangements and chromosomes according to the chromosome field. Hereditas 93: 146.

Raman, V.S. \& Kesavan, P.C. 1964. Meiosis and the nature of polyploidy in Turnera ulmifolia. J. Indian Bot. Soc. 43: 495-497.

Romero Zarco, C. 1986. A new method for estimating karyotype asymmetry. Taxon 35: 526-530.

Shore, J. y Barrett, S. C. H. 1985. Genetics of distyly and homostyly in the T. ulmifolia complex (Turneraceae). Heredity 55: 167-174.

Stebbins, G. L. 1971. Chromosomal Evolution in Higher Plants. AdissonWesley Publishing Company. Massachusetts.

Urban, I. 1883. Monographie der familie der Turneraceen. Jahrb. Königl. Bot. Gart. Berlin 2: 1-152. 